\title{
Inhibition of 12/15-Lipoxygenase Reduces Renal Inflammation and Injury in Streptozotocin-Induced Diabetic Mice
}

\author{
Jessica Faulkner', Chelsey Pye ${ }^{1}$, Mohamed Al-Shabrawey ${ }^{1,2}$ and Ahmed A Elmarakby ${ }^{1,3^{*}}$ \\ ${ }^{1}$ Department of Oral Biology, Georgia Regents University, Augusta, GA, USA \\ ${ }^{2}$ Department of Ophthalmology and Culver Vision Discovery Institute, Georgia Regents University, Augusta, GA, USA \\ ${ }^{3}$ Department of Pharmacology \& Toxicology, Georgia Regents University, Augusta, GA, USA
}

\begin{abstract}
Previous studies suggest that $12 / 15$ lipoxygenase (12/15-LO) is implicated in diabetic vascular complications. We hypothesize that 12/15-LO inhibition attenuates renal inflammation and injury in streptozotocin-induced diabetes. Diabetes was induced in wild-type C57BL/6J (WT) and 12/15-LO deficient mice using streptozotocin. Additionally, four groups of WT mice were also used; control non diabetic, diabetic, diabetic treated with the 12/15-LO inhibitor baicalein for 10 weeks and diabetic treated with baicalein only for the last 4 weeks of the experiment. After 10 weeks of induction of diabetes with streptozotocin, WT diabetic mice exhibited marked elevation in proteinuria together with elevation in the excretion levels of thiobarbituric acid reactive substance (TBARs), a marker of oxidative stress, and monocyte chemoattractant protein-1 (MCP-1), a marker of inflammation and these changes were significantly reduced in 12/15-LO deficient diabetic mice $(P<0.05)$. Similarly, pharmacological inhibition of 12/15-LO with baicalein prevented the elevation in renal 12-HETE production, the major murine metabolic product of 12/15-LO, in diabetic mice, and this effect was associated with decreased proteinuria, TBARs excretion and renal collagen deposition compared to untreated diabetic mice. Interestingly, the protective effects of baicalein were not noticed when only administered in the last 4 weeks of diabetes compared to untreated diabetic mice. WT diabetic mice displayed elevation in renal interleukin-6 (IL-6) levels and these changes were only reduced in diabetic mice treated with baicalein for 10 weeks $(P<0.05)$. The anti-inflammatory effects of baicalein or $12 / 15$-LO deficiency were further confirmed in lipopolysaccharide (LPS)-induced acute renal inflammation as inhibition of 12/15-LO reduced the elevation in renal soluble epoxide hydrolase expression in LPS-injected mice. These results suggest that increased 12/15-LO activity and 12-HETE production contribute to the elevation of renal oxidative stress, inflammation and injury in streptozotocin-induced diabetic mice.
\end{abstract}

Keywords: Diabetes; 12/15-LO Deficient mice; Baicalein; Proteinuria; Kidney; Oxidative stress; Inflammation

\section{Introduction}

The World Health Organization estimates that diabetes causes approximately five percent mortality worldwide and also expects that number to double before the year 2030 without drastic changes in the treatment options. Up to twenty percent of death is a result of end-stage renal disease, which is preceded by diabetic nephropathy (DN) [1]. The progression of DN manifests itself symptomatically via impairment in the glomerular filtration barrier resulting in microalbuminuria. Consequently, this evolves into more devastating complications including hypertension, progressive injury of the glomerular structure and thickening of the glomerular basement membrane, increased collagen deposition and macroalbuminuria [2-4]. Proper control of blood glucose levels reduces the risk of DN; however, new therapeutic strategies are required to prevent the incidence of early diabetic renal injury and counteract the expected progression to end-stage renal disease and dialysis among the rapidly expanding diabetic population.

Inflammation and oxidative stress are vital players in the pathogenesis of diabetic renal injury [5,6]. The elevation in renal macrophage infiltration is associated with changes in glomerular filtration rate together with increased intercellular adhesion molecule-1 (ICAM-1) and MCP-1 to direct their attachment to the site of inflammation $[7,8]$. Transforming Growth Factor- $\beta$ (TGF- $\beta$ ) and interleukins, such as interleukin-6 (IL-6), are subsequently transcribed in correlation with the macrophage levels stimulating inflammatory and fibrotic responses in the kidney [9-11]. In addition, the resulting release of reactive oxygen species (ROS) further exacerbates inflammatory process via increased renal apoptosis and injury [9]. It would fall to logic, therefore, that interference in these processes will attenuate the progression of renal injury in diabetic patients.

Eicosanoids are arachidonic acid metabolites that are known to be involved in the progression of inflammation in cardiovascular disease [12]. Phospholipase A2 enzyme metabolizes phospholipid to arachidonic acid, which is further metabolized via diverse pathways, including the cyclooxygenase (COX), lipoxygenase (LO), and cytochrome $\mathrm{P} 450$ pathways. The metabolic products of COX, prostaglandins and the metabolic products of the LO, leukotrienes, and hydroxyeicosatetraenoic acids (HETEs) are known to exacerbate inflammation [12]. Lipoxygenases (LOs) are a family of ironcontaining enzymes that mainly oxidize arachidonic acid to produce hydroxyeicosatetraenoic acids (HETEs). LOs are classified as 5-, 8-, $12-$, and 15-LOs according to the carbon atom of arachidonic acid at which oxygen is inserted. There are three major isoforms of 12-LO; platelet-type 12-LO, macrophage- or leukocyte-type 12-LO (12/15-

*Corresponding author: Ahmed A Elmarakby, Department of Oral Biology, Pharmacology \& Toxicology, Georgia Regents University, Augusta, GA., 30912 USA, Tel: 706-721-2748; Fax: 706-721-6252; E-mail: aelmarakby@gru.edu

Received April 03, 2015; Accepted May 25, 2015; Published May 30, 2015

Citation: Faulkner J, Pye C, Shabrawey M, Elmarakby AA (2015) Inhibition of 12/15-Lipoxygenase Reduces Renal Inflammation and Injury in StreptozotocinInduced Diabetic Mice. J Diabetes Metab 6: 555. doi:10.4172/2155-6156.1000555

Copyright: @ 2015 Faulkner J, et al. This is an open-access article distributed under the terms of the Creative Commons Attribution License, which permits unrestricted use, distribution, and reproduction in any medium, provided the original author and source are credited. 
LO), and epidermal-type 12-LO [13]. Human and rabbit 15-LOs as well as the leukocyte-type 12-LO have high homology, and are classified as 12/15-LO since they can form both 12-HETE and 15-HETE from arachidonic acid [13]. In the kidney, the leukocyte-type 12/15-LO activity predominates to synthesize 12 - and 15-HETE which mediate growth factor effects in vascular smooth muscle cells, fibroblasts, and mesangial cells, as well as responses to vascular injury $[12,13]$. The major product of 12-LO metabolism of arachidonic acid, 12-HETE has a role in various biological processes, including atherogenesis, cancer cell growth, and neuronal apoptosis [14-16]. In addition, 12-HETE has pro-inflammatory effects and has been implicated in diabetic vascular complication $[17,18]$. For example, high glucose-induced 12-HETE production in vascular endothelial and smooth muscle cells is linked to leukostasis via an intracellular adhesion molecule-1 dependent pathway $[12,19,20]$. 12-LO mediated-12-HETE production is also elevated in glomeruli of diabetic rats [17]. The flavonoid 12/15-LO specific inhibitor 5,6,7-trihydroxyflavone (baicalein), a Chinese herbal medicine, has been shown to reduce oxidative stress and inflammation in both diabetic and non-diabetic studies [21-23]. However, the renoprotective effects of baicalein in diabetes-induced renal injury have not been clearly elucidated. Our aim for this study was to utilize streptozotocin-induced type 1 diabetes in mice to determine whether pharmacological inhibition or gene deficiency of 12/15-LO hinders the progression of renal injury in diabetic mice via decreased renal oxidative stress and inflammation.

\section{Materials and Methods}

Wild-type C57BL/6J mice and 12/15-LO deficient mice (B6.129S2Alox $15^{\text {tm1Fun }} / \mathrm{J}$ ) were obtained from The Jackson Laboratory (Bar Harbor, ME). Backcrossing of 12/15-LO deficient mice with wildtype C57BL/6J, DNA extraction, and genotyping were performed according to the protocol provided by The Jackson Laboratory using PCR. All procedures with animals were performed in accordance with the Public Health Service Guide for the Care and Use of Laboratory Animals (Department of Health, Education, and Welfare publication, NIH 80-23) and Georgia Regents University guidelines. Mice were injected at 10-12 week of age with streptozotocin at a dose of $80 \mathrm{mg} /$ $\mathrm{kg}$ daily for three consecutive days to induce diabetes. Four to six days later, urine glucose was tested in streptozotocin injected mice using clinical glucose test strips (Bayer Corporation, Elkhart, IN) and only those with a reading of $300 \mathrm{mg} / \mathrm{dl}$ or higher were considered "diabetic". Control and diabetic WT and 12/15-LO deficient mice were used in this study ( $n=6-8 /$ group). Additionally, four groups of WT mice were used; control, diabetic, diabetic treated with 12/15-LO inhibitor baicalein $(100 \mathrm{mg} / \mathrm{kg} /$ day in drinking water, Cayman Chemical, Ann Arbor, MI) for 10 weeks as shown previously [12] and diabetic treated with baicalein only for the last four weeks of the experiment. After 10 weeks, mice were placed in metabolic cages for 24 hour urine collection before being terminated for blood and tissue collection. Blood glucose was obtained using a standard glucometer. Tissues and plasma were harvested and frozen.

\section{Renal injury}

Urinary protein excretions were measured as an index of renal injury using the Bradford Method for protein determination (BioRad, Hercules, CA). Kidney sections were embedded and frozen in Optimal Cutting Temperature (Tissue-Tek, Hatfield, PA). Kidneys were then sliced into $5-\mu \mathrm{m}$ sections and stained with Hematoxylin and Eosin ( $\mathrm{H} \& \mathrm{E})$ staining for detection of any histopathological changes and Masson's trichrome for collagen deposition according to the manufacturer's recommended protocols (Richard Allan Scientific, Kalamazoo, MI). Ten images were taken per mouse at $200 \mathrm{X}$ magnification power and intensity of the staining was scored blindly on a scale of 1 to 10 . Activity of the 12/15-LO was evaluated by LC/MS to measure the amount of renal 12-HETE, the major metabolic product of $12 / 15$-LO in mice, as we previously described [12].

\section{Renal oxidative stress and inflammation}

Urinary and plasma Thiobarbituric Acid Reactive Substances (TBARs) (Cayman Chemical, Ann Arbor, MI) and urinary monocyte chemotactic protein-1 (MCP-1) (BD Biosciences, San Jose, CA) excretion levels were assessed as markers of oxidative stress and inflammation, respectively. Furthermore, Renal MCP-1, IL-6, ICAM-1 and VCAM-1 levels were analyzed by Aushon Bio systems (Billerica, MA). Briefly, samples are incubated for three hours on the array plates that are pre-spotted with capture antibodies specific for each protein biomarker. Plates are decanted and washed four times before adding a cocktail of biotinylated detection antibodies to each well. After incubating with detection antibodies for 30 minutes, plates are washed four times and incubated for 30 minutes with streptavidin-horseradish peroxidase conjugate. All incubations are done at room temperature with shaking at $200 \mathrm{rpm}$. Plates are again washed four times before adding a chemiluminescent substrate. The plates are immediately imaged using the Aushon CCD imaging system, and data is analyzed using Aushon Array Analyst software. The amount of luminescent signal is proportional to the amount of each protein present in the original standard or sample. Concentrations are interpolated from a standard curve.

\section{Effect of 12/15-LO inhibition on acute renal inflammation}

To determine whether inhibition of $12 / 15-\mathrm{LO}$ affects renal expression of arachidonic acid metabolizing enzyme to provide renal protection in acute model of renal injury, additional groups of WT, with or without baicalein treatment for two weeks $(100 \mathrm{mg} / \mathrm{kg} / \mathrm{day}$ in drinking water), and 12/15-LO deficient mice were injected with lipopolysaccharide (LPS) from Salmonella typhimurium $(0.1 \mathrm{mg} /$ $\mathrm{kg}$; Sigma) to induce acute renal vascular inflammation ( $\mathrm{n}=4$ /group). Kidneys were collected and processed for analysis of COX2, CYP2J, CYP4A and sEH expression by Western blot 24 hours later.

\section{Western blotting for protein expression assessment}

Kidney was homogenized for Western Blotting utilizing RIPA homogenization buffer supplemented with protease and phosphatase inhibitor cocktails (Sigma, St Louis, MO). Samples were measured for protein concentration using the Bicinchoninic Acid method (Pierce, Rockford, IL) and supplemented with 2-mercaptoethanol and water appropriately to ensure equal protein $(30-50 \mu \mathrm{g})$ loading into $12 \%$ SDS-PAGE gels. The gels were electrophoretically run and transferred onto nitrocellulose membranes. Non-specific binding sites were blocked by incubating the blots overnight at $4^{\circ} \mathrm{C}$ in a Tris $\mathrm{NaCl}$ buffer (TBS) containing 5\% non-fat dry milk and $0.1 \%$ Tween 20 . The primary antibodies used were rabbit 12-LO (Abcam, Cambridge, MA), sEH, CYP4A (Santa Cruz Biotechnology, Santa Cruz, CA), CYP2J (Pierce, Rockford, IL), mouse COX2 (Cayman Chemical, Ann Arbor, MI) and ICAM-1 (R\&D Systems, Minneapolis, MN). The blots were then washed in a TBS- $0.1 \%$ Tween and incubated with the secondary antibody goat anti-rabbit- (1:5000) or goat anti-mouse-conjugated to horseradish peroxidase for 1 hour. Detection was accomplished using enhanced chemiluminescence Western blotting and band intensity was 
Citation: Faulkner J, Pye C, Shabrawey M, Elmarakby AA (2015) Inhibition of 12/15-Lipoxygenase Reduces Renal Inflammation and Injury in Streptozotocin-Induced Diabetic Mice. J Diabetes Metab 6: 555. doi:10.4172/2155-6156.1000555

measured densitometrically and the values were normalized to $\beta$-actin (Sigma, St Louis, MO).

\section{Data analysis}

Using Prism software (Graph Pad, San Diego, CA, USA), results were analyzed as means \pm SEM and were evaluated with one-way analysis of variance (ANOVA) followed by Tukey's post-hoc test for comparison of groups; any data succeeding with $\mathrm{p}<0.05$ was considered significant.

\section{Results}

\section{Blood glucose, body weight gain, food and water intake, urine excretion}

The streptozotocin-injected WT mice displayed a decrease $(\sim 18 \%$; $\mathrm{p}<0.05)$ in body weight ten weeks after induction of diabetes compared to their control counterparts with a modest non-significant increase in food intake. Induction of hyperglycemia with streptozotocin injection in WT mice increased water intake and urine volume compared to control mice. Although baicalein treatment, both at 4 weeks as well as 10 weeks, reduced water intake and urine output in WT diabetic mice, these changes were not significant. Blood pressure was not affected by either diabetes or baicalein treatment. Similarly, streptozotocin decreased body weight gain, water consumption and urine output in 12/15-LO deficient vs. control mice to the same extent in WT mice. There was a significant increase in non-fasting blood glucose level in WT diabetic mice compared to control (475 \pm 50 vs. $238 \pm 17 \mathrm{mg} / \mathrm{dl}$, $\mathrm{P}<0.05)$. Baicalein treatment for 4 weeks or 10 weeks did not reduce plasma glucose levels in diabetic mice compared to the untreated diabetic group (499 \pm 40 and $564 \pm 11 \mathrm{mg} / \mathrm{dl}$, respectively).

\section{Urinary markers of renal injury}

The role of 12/15-LO in the incidence of diabetic renal injury, oxidative stress and inflammation was initially assessed in 12/15LO deficient mice. As shown in Figure 1, induction of diabetes with streptrozotocin in WT mice produced marked elevation in proteinuria, the oxidative stress marker TBARs excretion and renal ICAM-1 expression as a marker of renal inflammation when compared with control WT $(\mathrm{P}<0.05)$. The increase in proteinuria, TBARs excretion and ICAM-1 expression were significantly reduced in diabetic 12/15-LO deficient vs. diabetic WT mice (Figure 1). Similarly, pharmacological inhibition of $12 / 15-\mathrm{LO}$ with baicalein significantly reduced the elevation in 12-LO activity as manifested by decreased levels of renal 12-LO expression and 12-HETE, the major murine metabolic product of $12 / 15-\mathrm{LO}$, in diabetic WT mice when compared to control (Figure $2 \mathrm{~A}$ and $2 \mathrm{~B}$, respectively). Induction of diabetes with streptozotocin significantly increased TBARs and MCP-1 excretion levels versus control (Figure $3 \mathrm{~A}$ and $3 \mathrm{~B}$ ). Baicalein-induced reduction in renal 12/15-LO expression and 12-HETE levels in diabetic WT mice (Figure $2 \mathrm{~A}$ and $2 \mathrm{~B}$ ) was associated with a significant reduction in TBARs excretion only in diabetic mice treated with baicalein for 10 weeks (Figure $3 \mathrm{~A}, \mathrm{P}<0.05$ ). However, the reduction in oxidative stress in baicalein treated diabetic mice for 10 weeks was not associated with reduction in renal MCP-1 excretion when compared to control (Figure 3B). Similarly plasma TBARs levels, an indicative of systemic oxidative stress, were significantly elevated in diabetic versus control ( $21 \pm 5$ vs. $10 \pm 1 \mathrm{nM}$ ) and was only reduced in baicalein treated diabetic mice for 10 weeks $(13 \pm 4 \mathrm{nM}, \mathrm{P}<0.05)$. Although baicalein treatment reduced the elevation in proteinuria in diabetic mice, this effect was only

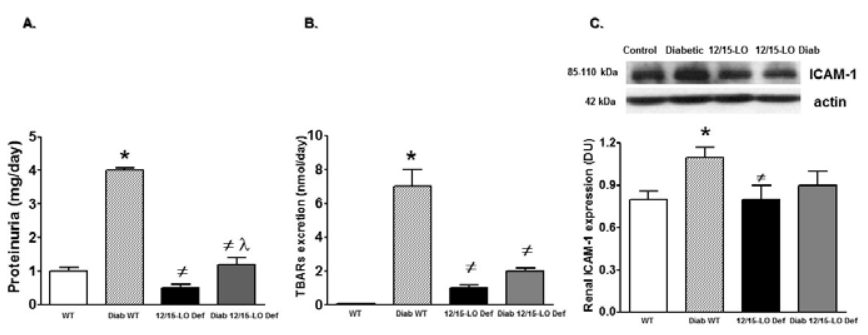

Figure 1: Urinary protein excretion (A), TBARs excretion (B) and renal ICAM1 expression levels relative to $\beta$-actin (C) in WT and 12/15-LO deficient mice after 10 weeks of induction of diabetes with streptozotocin. Diabetic WT mice displayed significant elevations in proteinuria, TBARs excretion and renal ICAM-1 expression compared to control. 12/15-LO deficiency prevented the elevation in proteinuria, TBARs excretion and renal ICAM-1 during diabetes. ( ${ }^{*}<<0.05$ vs. control WT mice, \#P<0.05 vs. diabetic WT mice and ${ }^{\wedge} \mathrm{P}<0.05$ vs. control 12/15-LO deficient mice, $n=6-8$ /group for excretion data and $n=4$ for ICAM-1 expression).
A.

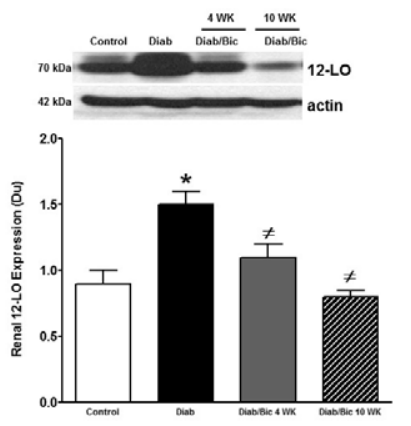

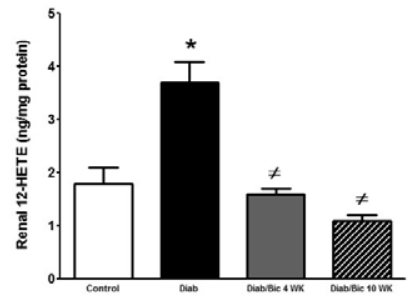

Figure 2: Renal 12-LO expression relative to $\beta$-actin $(A)$ and renal 12-HETE levels in control and diabetic WT mice with or without baicalein treatment for 4 and 10 weeks. Renal 12-LO expression and 12-HETE levels increased significantly in diabetic versus control mice. Inhibition of 12/15-LO with baicalein treatment resulted in significant reduction in 12-LO expression and $12-$ HETE levels either after 4 or 10 weeks $\left(\left({ }^{*} \mathrm{P}<0.05\right.\right.$ vs. control and $\# \mathrm{P}<0.05$ vs. diabetic mice, $n=6 /$ group).
A.

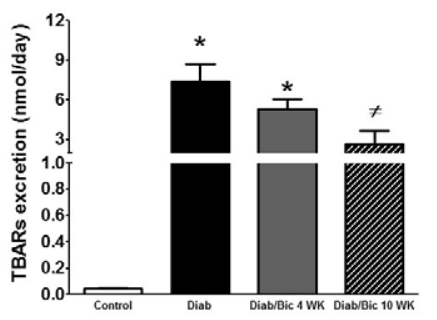

B.

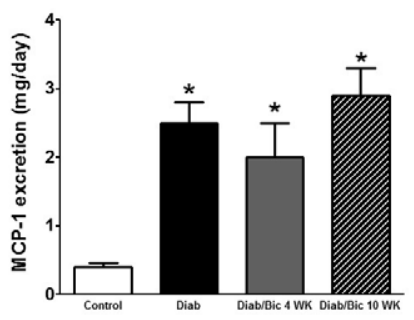

Figure 3: Urinary TBARs excretion (A) and MCP-1 excretion (B) in streptozotocin-induced diabetic mice. Both urinary TBARs and MCP-1 excretion levels were significantly elevated in diabetic versus control mice $\left({ }^{*} \mathrm{P}<0.05 \mathrm{vs}\right.$. control mice). Only baicalein treatment for 10 weeks significantly reduced TBARs excretion in diabetic mice (\#P<0.05 vs. diabetic mice, $n=6-8$ / group).

significant only in baicalein treated diabetic mice for 10 weeks (Figure 4C). Histologically, diabetic mice displayed glomerular hypertrophy, glomerular hypercellularity (H \& E staining, Figure 4A) as well as collagen deposition (Masson Trichrome staining, Figure 4B and 4D) 

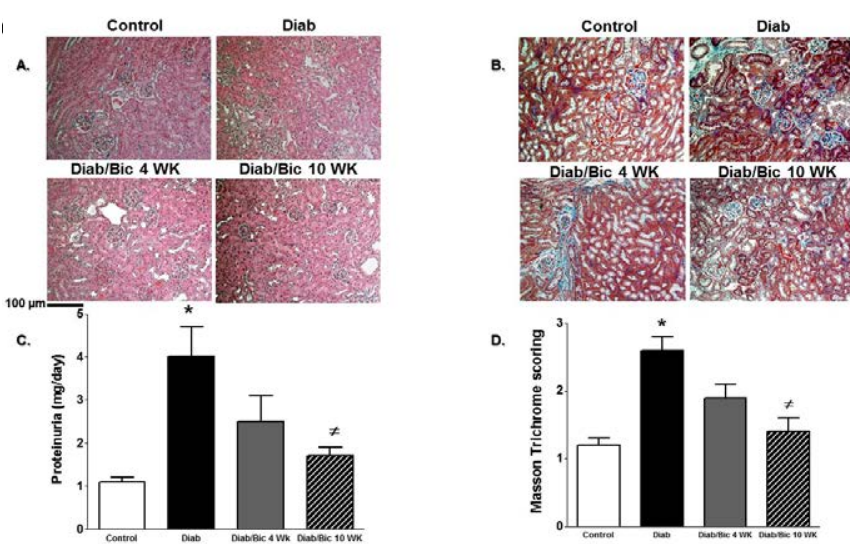

Figure 4: Representative images (200X) of kidney sections H \& E staining (A) and Masson's trichrome staining (blue staining, B), proteinuria (C) and average score for Masson's trichrome staining (D) in control and diabetic mice with or without baicalein treatment for 4 and 10 weeks. $\left({ }^{*} P<0.05\right.$ vs. control mice and \#P<0.05 vs. diabetic mice; $n=4 /$ group for histology and $n=6$ 8/group for proteinuria).
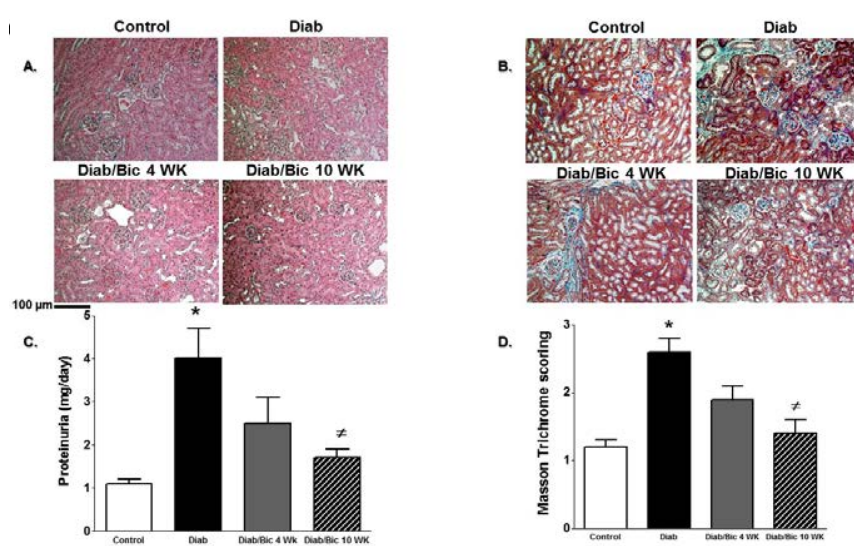

Figure 5: Effect of 12/15-LO inhibition with baicalein on renal inflammatory cytokines levels in streptozotocin-induced diabetic mice. Renal IL-6, MCP1 , VCAM-1 and ICAM-1 levels significantly increased in diabetic compared to control mice $\left({ }^{*} \mathrm{P}<0.05\right.$ vs. control mice). Only inhibition of $12 / 15$-LO with baicalein treatment for 10 weeks significantly reduced renal IL-6 levels in diabetic mice (\#P<0.05 vs. diabetic mice, $n=6 /$ group).

and this effect was reduced by baicalein treatment. Furthermore, only 10 weeks of baicalein treatment significantly reduced collagen deposition in diabetic mice (Figure 4D).

Inflammation plays a considerable role in the kidney in the development of diabetic renal injury [5,6]; therefore, we further quantify levels of inflammatory cytokines in the kidney of diabetic mice. Induction of diabetes with streptozotocin significantly increased renal levels of IL-6, MCP-1, ICAM-1 and VCAM-1 compared to control WT mice (Figure 5, P0.05). Although baicalein tended to reduced levels of renal inflammatory cytokines in diabetic mice, only 10 weeks of baicalein treatment significantly reduced renal IL-6 levels in diabetic mice (Figure 5) when compared to control group.

Effect of 12/15-LO inhibition on arachidonic acid metabolizing enzymes in the kidney of LPS-injected mice

Induction of diabetes with streptozotocin for 10 weeks up- regulate $\mathrm{COX}-2,12-\mathrm{LO}$ derived inflammatory prostaglandins and 12-HETE production, respectively and down-regulate CYP2J derived anti-inflammatory EETs production in the kidney suggesting a cross-talk between arachidonic acid metabolizing enzymes to trigger renal inflammation in diabetes (data not shown). Because baicalein treatment reduced COX-2 and 12-LO expression and improved CYP2J expression in diabetic kidneys, we further determined whether baicalein or 12/15-LO deficiency would provide similar pattern on arachidonic acid metabolizing enzymes in acute LPS-induced renal inflammation. Renal expression levels of COX2, epoxygenase, hydroxylase, and soluble epoxide hydrolase were assessed after 24 hours of LPS injection in WT mice, with or without baicalein treatment for two weeks prior to LPS injection, and 12/15-LO deficient mice. COX2 catalyzes the production of the inflammatory prostaglandins, hydroxylase catalyzes the production of the inflammatory metabolites 20-HETE, epoxygenase catalyzes the production of the anti-inflammatory epoxyeicosatrienoic acids (EETs) whereas soluble epoxide hydrolase (sEH) limits EETs availability by metabolizing them to inactive metabolites [24]. Renal expression level of 12-LO was lesser in 12/15-LO deficient mice versus WT control (Figure 6A). Renal 12-LO expression was significantly elevated in LPS injected WT mice and was reduced in either baicalein treated LPS injected mice or LPS injected 12/15-LO deficient mice $(\mathrm{P}<0.05$, Figure 6A). Basal renal COX2 levels were lesser in $12 / 15$ LO deficient mice than WT mice (Figure 6B). Although renal COX2 expression did not change in LPS injected WT mice, baicalein treatment lowered COX2 expression in LPS WT injected mice (Figure 6B). Surprisingly, injection of LPS in 12/15-LO deficient mice increased renal COX2 expression compared to 12/15-LO deficient control (Figure 6B). LPS injection also significantly increased expression of renal hydroxylase CYP4A in WT mice compared to control and this effect was reduced in 12/15-LO deficient mice with or without LPS injection (Figure 7A). Although There was no difference in renal CYP2J epoxygenase in WT and 12/15-LO deficient mice even after LPS treatment (Figure 7B), injection of LPS was associated with elevation of renal $\mathrm{sEH}$, the epoxyeicosatrienoic acid metabolizing enzyme, in WT mice (Figure $7 \mathrm{C}, \mathrm{P}<0.05$ ) and renal $\mathrm{sEH}$ expression was significantly reduced in 12/15-LO deficient mice with or without LPS injection.

\section{Discussion}

The potential use of herbal medicine in combating many
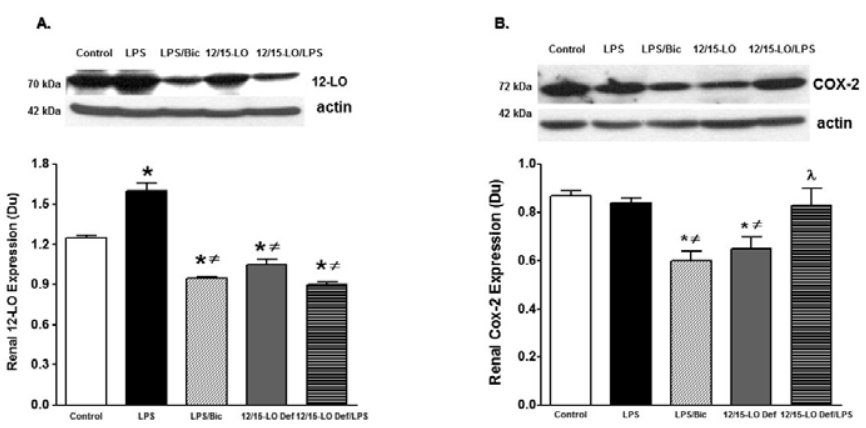

Figure 6: Renal 12-LO (A) and COX2 (B) expression levels relative to $\beta$-actin in WT with or without baicalein treatment and 12/15-LO deficient mice after 24 hours of LPS injection. Renal 12-LO expression significantly increased in LPS injected mice compared with control mice and was reduced in baicalein treated WT mice or in 12/15-LO deficient mice. Renal COX2 expression was significantly reduced in baicalein treated LPS injected WT mice versus control. Renal COX2 expression was lesser in 12/15-LO deficient mice versus control and increased after LPS injection ( ${ }^{*} P<0.05$ vs. control mice, $\# P<0.05$ vs. LPS injected WT mice, and ${ }^{\wedge} P<0.05$ vs. control $12 / 15$-LO deficient mice, $n=4 /$ group). 

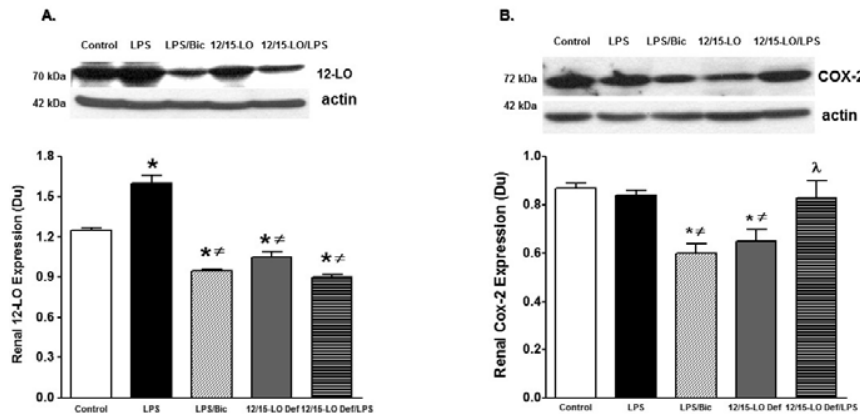

Figure 7: A. Renal CYP4A hydroylase (A), CYP2J epoxygenase (B) and soluble epoxide hydrolase ( $\mathrm{sEH}, \mathrm{C}$ ) expression levels relative to $\beta$-actin in WT with or without baicalein treatment and 12/15-LO deficient mice after 24 hours of injection with LPS. Renal CYP4A hydroxylase was significantly elevated in LPS injected WT mice versus control and was reduced in LPS injected 12/15-LO deficient mice. Although there was no difference in renal CYP2J epoxygenase among mice group, renal sEH increased in LPS injected WT mice. Baicalein treatment or 12/15-LO deficiency reduced the elevation in renal $\mathrm{SEH}$ expression in LPS injected WT mice $\left({ }^{*} \mathrm{P}<0.05\right.$ vs. control mice, \#P $<0.05$ vs. LPS injected WT mice, and ${ }^{\wedge} P<0.05$ vs. control 12/15-LO deficient mice, $n=4 /$ group)

diseases, such as cancer Alzheimer, viral and bacterial infections and cardiovascular disease, has attracted many investigator nowadays. Baicalein, is a flavonoid isolated from the traditional Chinese herbal medicine with multiple biological activities, such as anti-inflammatory and anti-oxidant through free radical scavenging and lipid peroxidase inhibiting properties $[22,25,26]$. Baicalein has been also used as an inhibitor of $12 / 15$-LO [23]. Studies have shown that $12 / 15-\mathrm{LO}$ is upregulated to increase 12- and 15-HETEs production which could exacerbate inflammation and oxidative stress in diabetes to perturb the progression of organ damage [13,17]. In diabetes, targeting early features of renal injury could prevent the progression of DN and endstage renal disease [5,9]. Accordingly, the current study demonstrates that 10 week diabetic mice showed a marked proteinuria together with early histopathological changes of diabetic renal injury such as proteinuria, glomerular hypertrophy and hyper cellularity and collagen deposition. The early signs of diabetic renal injury were associated with up-regulation of renal 12-LO expression and increased 12-HETE production; the major product of $12 / 15-\mathrm{LO}$ in rodents, as well as increased renal oxidative stress and inflammation. The elevation in proteinuria, renal oxidative stress and inflammation were significantly reduced in 12/15-LO deficient diabetic mice. Similarly pharmacological inhibition of 12/15-LO with baicalein treatment for 10 weeks after induction of diabetes reduced elevation in proteinuria, oxidative stress and renal IL-6 levels in diabetic mice whereas treating 6 weeks diabetic mice with baicalein for 4 more weeks failed to lower proteinuria, renal injury and oxidative stress despite reducing renal 12-LO expression and 12-HETE production. Taken together our data suggest a role of 12/15$\mathrm{LO}$ in the incidence of diabetic renal injury probably via increased oxidative stress and cytokines mediated inflammation.

Diabetic renal injury is characterized by a marked degree of protein- and albuminuria and histologically by glomerular basement membranes thickness, glomerular hypertrophy and mesangial extracellular matrix expansion [17,27]. These changes mainly occur due to hyperglycemia, oxidant stress, and increase cytokines production $[17,28]$. In the kidney, the leukocyte-type 12/15-LO activity predominates to synthesize 12 - and 15-HETE which mediate growth factor effects in vascular smooth muscle cells, fibroblasts, and mesangial cells, as well as responses to vascular injury [29,30]. Urinary excretion of 12-HETE were elevated in diabetic versus control subjects [31]. In vitro, high-glucose increased 12/15-LO activity and expression in mesangial, podocytes and endothelial cell [32-34]. The increased in $12 / 15$-LO in high glucose treated podocyte was also associated with elevation in collagen and phospho-p38 mitogen activated protein kinase (MAPK) protein expression and pharmacological inhibition of 12/15LO prevented these changes [35]. In streptozotocin-induced diabetic rats, elevated renal 12/15-LO levels was associated with increased renal fibronectin and collagen expression [32,35]. Pharmacological inhibition of 12-LO modulated renal injury in streptozotocin-induced type 1 diabetic rats [17]. Mice received 12/15-LO siRNA showed a significant reduction in albuminuria, kidney-to-body weight ratios, glomerular mesangial matrix expansion, renal structural damage, and monocyte/macrophage infiltration [13] suggesting an important role of $12 / 15-\mathrm{LO}$ pathway activation in extracellular mesangial matrix and collagen deposition during the development of diabetic renal injury. Consistent with previous findings, the current study suggest that genetic manipulation as well as pharmacological inhibition of 12/15-LO with baicalein treatment for 10 weeks significantly reduced renal collagen deposition and proteinuria demonstrating a clear-cut relationship between activation of the renal 12/15-LO pathway and the development of proteinuria. The failure of 4 weeks of baicalein treatment to reduce collagen deposition and proteinuria could be attributed to establishment of marked degree of proteinuria and extracellular matrix deposition after 6 weeks of induction of diabetes with streptozotocin that was hard to be off-sited using baicalein only in the last 4 weeks of the experiment.

Renal infiltration and activation of monocytes/macrophages and its associated inflammation have been implicated in the pathogenesis of DN [5-8]. MCP-1, a chemokine that recruits activated monocytes to sites of injury and inflammation, is enhanced in diabetic glomeruli and contributes to collagen deposition in DN [7-8]. Increased MCP-1 excretion level correlates with the elevation in proteinuria in diabetes [36]. Increasing evidence also indicates a role for various inflammatory molecules, including chemokines, adhesion molecules, and proinflammatory cytokines, in diabetic complications [8,37]. Previous studies show that $12 / 15$ - LO and its products can induce inflammatory genes, including MCP-1 and IL-6 [37,38]. The 12/15-LO siRNAs significantly decreased MCP-1 expression and macrophage infiltration in the diabetic kidney [13]. Although neither 4 nor 10 weeks of baicalein treatment reduced the elevation in MCP-1 excretion in diabetic mice in our study, the decrease in proteinuria in diabetic 12/15-LO deficient mice and 10 week baicalein treated diabetic mice was associated with decreased renal ICAM-1 expression and renal IL-6 levels, respectively, when compared to diabetic WT mice suggesting that 12/15-LO could contribute to the progression of renal injury in diabetes via increased renal inflammation. The reduction in proteinuria in diabetic $12 / 15-\mathrm{LO}$ deficient mice did not seems to be attributed to significant decrease in blood glucose or blood pressure (data are not shown).

Oxidative stress is considered a causal link between elevated glucose levels and the development of diabetic renal injury $[39,40]$. 12-HETE treatment and up-regulation of 12/15-LO are known to increase oxidative stress in diabetes [12]. In diabetic mice lacking 12/15-LO, a significant decrease in retinal hyperpermeability was recently shown compared with diabetic WT mice [12,41]. Additionally, the permeability effect of 12-HETE was significantly reduced in mice lacking the catalytic subunit of NADPH oxidase, the major vascular source of superoxide production, (NOX2) compared with the WT mice suggesting that $12 / 15-\mathrm{LO}$ is implicated in diabetic retinopathy via 
NADPH dependent mechanism [12,41]. In our study, streptozotocininduced diabetic renal injury was associated with elevation in lipid peroxidation as manifested with increased TBARs excretion. 12/15LO deficiency or inhibition of $12 / 15-\mathrm{LO}$ with baicalein prevented the elevation in lipid peroxidation in diabetes; however, 4 weeks of baicalein treatment failed to significantly lower lipid peroxidation after 6 weeks of induction of diabetes probably due to marked establishment of high levels of lipid peroxidation. It is noteworthy to mention that TBARs excretion levels were higher in non-diabetic 12/15-LO deficient mice compared to WT mice. We postulate that 12/15-LO deficiency might compensatory up-regulate other arachidonic acid producing inflammatory metabolites to mildly increase oxidative stress in $12 / 15$ deficient mice than WT control mice.

$12 / 15$-LO expression is also up-regulated to increase inflammation and vascular permeability during acute model of tissue injury such as LPS-induced acute lung injury [42-44]. The link between 12/15-LO up-regulation and the other arachidonic acid metabolizing enzymes is not clear during acute inflammation. Because baicalein treatment reduced the elevation in renal COX-2 and 12-LO expression and improved renal CYP2J expression levels in streptozotocin-induced diabetes (data not shown), we further determined whether baicalein or 12/15-LO deficiency would provide similar pattern on the expression levels of arachidonic acid metabolizing enzyme in acute LPS-induced renal inflammation. In our study, renal 12-LO expression were elevated after 24 hours of induction of acute inflammation using LPS injection in mice. The elevation in 12-LO was associated with increased expression of the CYP4A hydroxylase expression to increase inflammatory 20-HETE production and inflammatory sEH expression, which limits anti-inflammatory EETs by increasing their degradation [24]. Genetic manipulation as well as pharmacological inhibition 12/15-LO with baicalein reduced renal 12/15-LO expression after 24 hours of LPS injection and this effect was associated with decreased renal inflammatory CYP4A and sEH expression levels suggesting that 12/15-LO could cross-talk with other arachidonic acid metabolizing enzyme to boost inflammatory cascade during the establishment of acute inflammation

In summary, our data suggest that up-regulation of $12 / 15-\mathrm{LO}$ increases 12-HETE production, which could increase proteinuria via increase inflammatory cytokines production and oxidative stress in kidney of diabetic mice. Pharmacological inhibition or genetic manipulation of 12/15-LO attenuates diabetes-induced renal injury and kidney histopathological changes by reducing the oxidative stress and inflammatory cytokines production. Baicalein might have direct anti-inflammatory and antioxidant effects independent on 12/15LO inhibition [21,22] and inhibition of $12 / 15-\mathrm{LO}$ with baicalein was previously reported to reduce $12 / 15$-LO expression in rats subjected to middle cerebral artery occlusion [45]. Therefore, we suggest that reduction of 12-LO expression upon baicalein treatment both in diabetic and LPS injected mice could be attributed to reduced inflammation and oxidative stress. Contrary to the literature [46], the reno-protective effect of 12/15-LO inhibition does not seem to be mediated by improving insulin sensitivity. Furthermore, Pharmacological inhibition of 12/15-LO with baicalein not only reduced renal inflammatory 12LO expression but also improved anti-inflammatory EETs availability via decreased sEH expression in the mice kidney of acute LPS-induced renal inflammation. These observations are of clinical relevance as baicalein herbal supplementation could be a promising tool to prevent the progression of diabetic renal injury via inhibition of $12 / 15-\mathrm{LO}$ mediated elevation in oxidative stress and cytokines production given the fact that careful glycemic control in diabetic patients is hard to achieve despite current available therapeutic regimens.

\section{Acknowledgement}

The authors want to acknowledge Dr. Krishna Rao Maddipati at the Department of Pathology, Wayne State University, Detroit, MI for his assistance with 12-HETE analysis. This work was supported by grants from the Georgia Regents University Bridge funding grant and American Heart Association Scientist Development to $A$ Elmarakby and 1R01EY023315-01 award to M. Al-Shabrawey.

\section{References}

1. Park CW (2014) Diabetic kidney disease: from epidemiology to clinical perspectives. Diabetes Metab J 38: 252-260.

2. Maclsaac RJ, Ekinci El, Jerums G (2014) 'Progressive diabetic nephropathy How useful is microalbuminuria?: contra'. Kidney Int 86: 50-57.

3. Eilat-Tsanani S, Reitman A, Dayan M, Mualem Y, Shostak A (2014) Management of kidney disease in patients with diabetes in the primary care setting. Prim Care Diabetes 8: 159-163.

4. Jones RK, Hampton D, O'Sullivan DJ, Phillips AO (2013) Diabetes and rena disease: who does what? Clin Med 13: 460-464.

5. Duran-Salgado MB, Rubio-Guerra AF (2014) Diabetic nephropathy and inflammation. World J Diabetes 5: 393-398.

6. Forbes JM, Cooper ME (2013) Mechanisms of diabetic complications. Physiol Rev 93: 137-188.

7. Har R, Scholey JW, Daneman D, Mahmud FH, Dekker R, et al. (2013) The effect of renal hyperfiltration on urinary inflammatory cytokines/chemokines in patients with uncomplicated type 1 diabetes mellitus. Diabetologia 56: 11661173.

8. Okada S, Shikata K, Matsuda M, Ogawa D, Usui H, et al. (2003) Intercellular adhesion molecule-1-deficient mice are resistant against renal injury after induction of diabetes. Diabetes 52: 2586-2593.

9. Tesch GH (2010) Macrophages and diabetic nephropathy. Semin Nephrol 30 290-301.

10. Chow FY, Nikolic-Paterson DJ, Atkins RC, Tesch GH (2004) Macrophages in streptozotocin-induced diabetic nephropathy: Potential role in renal fibrosis. Nephrology, dialysis, transplantation : official publication of the European Dialysis and Transplant Association - European Renal Association 19: 29872996.

11. Lange-Sperandio B, Forbes MS, Thornhill B, Okusa MD, Linden J, et al. (2004) A2a adenosine receptor agonist and pde4 inhibition delays inflammation but fails to reduce injury in experimental obstructive nephropathy. Nephron Experimental nephrology 100: e113-e123.

12. Othman A, Ahmad S, Megyerdi S, Mussell R, Choksi K, et al. (2005) 12/15-lipoxygenase-derived lipid metabolites induce retinal endothelial cell barrier dysfunction: Contribution of nadph oxidase. PloS one 8: e57254.

13. Yuan H, Lanting L, Xu ZG, Li SL, Swiderski P, et al. (2008) Effects of cholesteroltagged small interfering rnas targeting 12/15-lipoxygenase on parameters of diabetic nephropathy in a mouse model of type 1 diabetes. Am J Physiol Renal Physiol 295: F605-617.

14. Kandouz M, Nie D, Pidgeon GP, Krishnamoorthy S, Maddipati KR, et al. (2003) Platelet-type 12-lipoxygenase activates nf-kappab in prostate cancer cells. Prostaglandins \& other lipid mediators 71: 189-204

15. Cabral M, Martín-Venegas R, Moreno JJ (2013) Role of arachidonic acid metabolites on the control of non-differentiated intestinal epithelial cell growth Int J Biochem Cell Biol 45: 1620-1628.

16. Chakrabarti SK, Wen $Y$, Dobrian AD, Cole BK, Ma Q, et al. (2011) Evidence for activation of inflammatory lipoxygenase pathways in visceral adipose tissue of obese zucker rats. Am J Physiol Endocrinol Metab 300: e175-e187.

17. Ma J, Natarajan R, LaPage J, Lanting L, Kim N, et al. (2005) 12/15-lipoxygenase inhibitors in diabetic nephropathy in the rat. Prostaglandins Leukot Essent Fatty Acids 72 : $13-20$

18. Xu ZG, Li SL, Lanting L, Kim YS, Shanmugam N, et al. (2006) Relationship between 12/15-lipoxygenase and COX-2 in mesangial cells: potential role in diabetic nephropathy. Kidney Int 69: 512-519.

19. Patricia MK, Kim JA, Harper CM, Shih PT, Berliner JA, et al. (1999) Lipoxygenase products increase monocyte adhesion to human aortic endothelial cells. Arteriosclerosis, thrombosis, and vascular biology 19: 2615-2622. 
Citation: Faulkner J, Pye C, Shabrawey M, Elmarakby AA (2015) Inhibition of 12/15-Lipoxygenase Reduces Renal Inflammation and Injury in Streptozotocin-Induced Diabetic Mice. J Diabetes Metab 6: 555. doi:10.4172/2155-6156.1000555

20. Natarajan R, Bai W, Lanting L, Gonzales N, Nadler J (1997) Effects of high glucose on vascular endothelial growth factor expression in vascular smooth muscle cells. Am J Physiol 273: H2224-2231.

21. Ahad A, Mujeeb M, Ahsan H, Siddiqui WA (2014) Prophylactic effect of baicalein against renal dysfunction in type 2 diabetic rats. Biochimie 106: 101-110.

22. Stavniichuk R, Drel VR, Shevalye H, Maksimchyk Y, Kuchmerovska TM, et al. (2011) Baicalein alleviates diabetic peripheral neuropathy through inhibition of oxidative-nitrosative stress and p38 mapk activation. Exp Neurol 230: 106-113.

23. Song L, Yang H, Wang HX, Tian C, Liu Y, et al. (2014) Inhibition of $12 / 15$ lipoxygenase by baicalein reduces myocardial ischemia/reperfusion injury via modulation of multiple signaling pathways. Apoptosis 19: 567-580.

24. Elmarakby AA (2012) Reno-protective mechanisms of epoxyeicosatrienoic acids in cardiovascular disease. American journal of physiology Regulatory, integrative and comparative physiology 302: R321-330.

25. Pu P, Wang XA, Salim M, Zhu LH, Wang L, et al. (2012) Baicalein, a natural product, selectively activating ampkalpha(2) and ameliorates metabolic disorder in diet-induced mice. Molecular and cellular endocrinology 362: 128-138.

26. Yang LP, Sun HL, Wu LM, Guo XJ, Dou HL, et al. (2009) Baicalein reduces inflammatory process in a rodent model of diabetic retinopathy. Invest Ophthalmol Vis Sci 50: 2319-2327.

27. Ibrahim HN, Hostetter TH (1997) Diabetic nephropathy. J Am Soc Nephrol 8: 487-493.

28. Wolf G, Ziyadeh FN (1999) Molecular mechanisms of diabetic renal hypertrophy Kidney Int 56: 393-405.

29. Ardaillou R, Baud L, Sraer J (1989) Lipoxygenase products and their functions in glomeruli. Adv Exp Med Biol 259: 49-74.

30. Jim K, Hassid A, Sun F, Dunn MJ (1982) Lipoxygenase activity in rat kidney glomeruli, glomerular epithelial cells, and cortical tubules. J Biol Chem 257 10294-10299.

31. Antonipillai I, Nadler J, Vu EJ, Bughi S, Natarajan R, et al. (1996) A 12-lipoxygenase product, 12-hydroxyeicosatetraenoic acid, is increased in diabetics with incipient and early renal disease. J Clin Endocrinol Metab 81: 1940-1945.

32. Kang SW, Adler SG, Nast CC, LaPage J, Gu JL, et al. (2001) 12-lipoxygenase is increased in glucose-stimulated mesangial cells and in experimental diabetic nephropathy. Kidney Int 59: 1354-1362.

33. Natarajan R, Nadler JL (2004) Lipid inflammatory mediators in diabetic vascular disease. Arterioscler Thromb Vasc Biol 24: 1542-1548.

34. Natarajan R, Gu JL, Rossi J, Gonzales N, Lanting L, et al. (1993) Elevated glucose and angiotensin ii increase 12-lipoxygenase activity and expression in porcine aortic smooth muscle cells. Proc Natl Acad Sci U S A 90: 4947-4951.
35. Kang SW, Natarajan R, Shahed A, Nast CC, LaPage J, et al. (2003) Role of 12-lipoxygenase in the stimulation of p38 mitogen-activated protein kinase and collagen alpha5(iv) in experimental diabetic nephropathy and in glucosestimulated podocytes. J Am Soc Nephrol 14: 3178-3187.

36. Morii T, Fujita H, Narita T, Shimotomai T, Fujishima H, et al. (2003) Association of monocyte chemoattractant protein-1 with renal tubular damage in diabetic nephropathy. J Diabetes Complications 17: 11-15.

37. Giunti S, Tesch GH, Pinach S, Burt DJ, Cooper ME, et al. (2008) Monocyte chemoattractant protein-1 has prosclerotic effects both in a mouse model of experimental diabetes and in vitro in human mesangial cells. Diabetologia 51: 198-207.

38. Wen Y, Gu J, Chakrabarti SK, Aylor K, Marshall J, et al. (2007) The role of 12/15-lipoxygenase in the expression of interleukin-6 and tumor necrosis factor-alpha in macrophages. Endocrinology 148: 1313-1322.

39. Lee DY, Wauquier F, Eid AA, Roman LJ, Ghosh-Choudhury G, et al. (2013) Nox4 nadph oxidase mediates peroxynitrite-dependent uncoupling of endothelial nitric-oxide synthase and fibronectin expression in response to angiotensin ii: Role of mitochondrial reactive oxygen species. J Biol Chem 288: 28668-28686.

40. Khazim K, Gorin Y, Cavaglieri RC, Abboud HE, Fanti P (2013) The antioxidant silybin prevents high glucose-induced oxidative stress and podocyte injury in vitro and in vivo. Am J Physiol Renal Physiol 305: F691-700.

41. Ibrahim AS, Elshafey S, Sellak H, Hussein KA, El-Sherbiny M, et al. (2015) A lipidomic screen of hyperglycemia-treated hrecs links 12/15-lipoxygenase to microvascular dysfunction during diabetic retinopathy via nadph oxidase. $J$ Lipid Res 56: 599-611.

42. Rossaint J, Nadler JL, Ley K, Zarbock A (2012) Eliminating or blocking $12 / 15$-lipoxygenase reduces neutrophil recruitment in mouse models of acute lung injury. Crit Care 16: R166.

43. Middleton MK, Zukas AM, Rubinstein T, Jacob M, Zhu P, et al. (2006) Identification of 12/15-lipoxygenase as a suppressor of myeloproliferative disease. J Exp Med 203: 2529-2540.

44. Zarbock A, Distasi MR, Smith E, Sanders JM, Kronke G, et al. (2009) Improved survival and reduced vascular permeability by eliminating or blocking 12/15-lipoxygenase in mouse models of acute lung injury (ALI). J Immunol 183: 4715-4722.

45. Cui L, Zhang X, Yang R, Liu L, Wang L, et al. (2010) Baicalein is neuroprotective in rat MCAO model: role of 12/15-lipoxygenase, mitogen-activated protein kinase and cytosolic phospholipase A2. Pharmacol Biochem Behav 96: 469475 .

46. Vahsen S, Rakowski K, Ledwig D, Dietze-Schroeder D, Swifka J, et al. (2006) Altered glut4 translocation in skeletal muscle of 12/15-lipoxygenase knockout mice. Hormone and metabolic research $=$ Hormon- und Stoffwechselforschung =Hormones et metabolisme 38: 391-396. 